\title{
Infección por Cyclospora sp. en tres pacientes inmunocompetentes
}

\author{
Piedad Agudelo, Marcos Restrepo, María Teresa Galvis, David Botero \\ Instituto Colombiano de Medicina Tropical, Medellin, Colombia
}

\begin{abstract}
Resumen
Se presentan tres casos de pacientes inmunocompetentes con síndrome diarreico que consultaron al Instituto Colombiano de Medicina Tropical en Medellín. El único agente patógeno aislado de sus muestras fecales fue Cyclospora sp. Una paciente fue tratada con un medicamento antiparasitario experimental, la nitazoxanida, y los otros dos pacientes con trimetroprim-sulfametoxazole, todos con buenos resultados. Se presenta una revisión de algunos aspectos de la enfermedad y se recomienda que, en casos de diarrea prolongada, se disponga de un protocolo completo para estudio de parásitos en materia fecal, además de capacitar muy bien al personal de salud que lee los coprológicos para establecer un diagnóstico diferencial entre el grupo de las coccidias.
\end{abstract}

Palabras clave: coccidia, Cyclospora, diarrea crónica.

\section{Cyclospora sp. infection in three immunocompetent patients}

\begin{abstract}
We describe three patients seen at the Instituto Colombiano de Medicina Tropical in Medellin, in whose case the only parasitic agent incriminated as the cause of prolonged diarrhea was the protozoan Cyclospora sp. One patient was treated with a new antiparasitic drug, nitazoxanide, and the other two with trimethoprim-sulfamethoxazole, all with good results. A revision of some aspects of the illness is presented. It is recommended that in cases of prolonged diarrhea, a complete protocol for the study of parasites in faeces be established and that the microscopists be trained to identify the coccidian pathogens.
\end{abstract}

Key words: coccidia, Cyclospora, chronic diarrhea.

La subclase Coccidia está localizada en la clase Sporozoa, con varios géneros y especies de parásitos, caracterizada por incluir protozoos con reproducción sexuada o asexuada, de donde resultan las esporogonias y, luego, los ooquistes de gran importancia en medicina veterinaria. Esta subclase, además de infectar al ser humano, puede infectar a los animales (1). El único orden de esta subclase que tiene importancia en la parasitología

Correspondencia: Apartado aéreo 52162, Medellin Colombia; fax: (574) 5716960 ,icmt@epm.net.co

Recibido: 26/11/99; aceptado: 28/01/00 médica es Eucoccidiida, la cual contiene parásitos tanto de animales vertebrados como de invertebrados. En este orden están incluidos los géneros /sospora, Criptosporidium y Cyclospora, que son protozoos de localización intestinal, los dos últimos considerados como 'nuevos' patógenos para el hombre aunque se identificaron hace más de quince años $(1,2)$.

En 1986, Soave y colaboradores informaron el hallazgo de unas estructuras no esporuladas que semejaban esporas de hongos o cuerpos de coccidias y que estaban relacionadas con diarrea crónica (3). En Perú, en 1989, Naranjo y 
colaboradores encontraron estas formas quísticas en las materias fecales de niños con diarrea y sugirieron que eran semejantes a quistes de Cryptosporidium muris (4). Otros investigadores observaron estas estructuras y las describieron como coccidias no esporuladas, algas azulverdosas, cuerpos similares a Cyanobacterium y Cryptosporidium grande (5-8).

En 1993, Ortega y colaboradores clasificaron este nuevo parásito en el género Cyclospora y, posteriormente, lo definieron como la especie Cyclospora cayetanensis $(9,10)$, al demostrar con el microscopio electrónico que los ooquistes tenian esporozoítos con organelos característicos de las coccidias, phylum Apicomplexa. La confirmación en el intestino se hizo al observar las formas intraepiteliales del parásito en su estadio asexual en las biopsias de intestino de los pacientes sintomáticos $(9,10)$. En 1996 , Relman y colaboradores determinaron la secuencia de ADN al hacer un análisis filogenético (11) y sugirieron que la especie Cyclospora asociada con el hombre, está más estrechamente relacionada con el género Eimeria que con el género Cryptosporidium y con otros géneros de coccidias, presentes tanto en pacientes inmunocompetentes como en inmunodeprimidos.

En 1996, Smith y colaboradores informaron la presencia de ooquistes semejantes a $C$. cayetanensis en la materia fecal de mandriles y monos chimpancés de Tanzania (12). Otros autores publicaron informes semejantes al examinar materias fecales de pollos en México (13), patos en Perú (14) y perros en Brasil (15). En 1999, Eberhard y colaboradores caracterizaron nuevas especies del género Cyclospora en monos -C. cercopitheci, C. colobi y C. papionis- por métodos morfológicos y moleculares (16).

El género Cyclospora encontrado en el ser humano tiene una distribución mundial, pues existen casos informados en muchos lugares: Nepal, Papua (Nueva Guinea), Indonesia, India, Pakistán, norte de Africa, Reino Unido, Caribe, Estados Unidos, México, Haití, Centroamérica y Suramérica. Se encuentra tanto en grupos de pacientes inmunocompetentes como en inmunodeprimidos (3-8,17-23).
En Colombia no se han encontrado casos informados de este parásito y, por tanto, se justifica esta publicación con el propósito de describir tres casos en pacientes inmunocompetentes con diarrea, en quienes se encontró como agente incriminado el protozoo Cyclospora sp. También se quiere llamar la atención sobre la importancia de buscar sistemáticamente este protozoo en pacientes con diarrea crónica.

\section{Materiales y métodos}

En la consulta médica del Instituto Colombiano de Medicina Tropical se tiene como norma que, en los pacientes con diarrea de más de una semana de duración, además del examen coprológico para búsqueda de parásitos intestinales, se haga una preparación para colorearla por el método de Ziehl-Neelsen modificado (9). Con este método se encontraron quistes ácido-resistentes que se estudiaron detenidamente por su tamaño. Se identificaron tres casos con estructuras semejantes a Cryptosporidium, pero de mayor tamaño.

El estudio parasitológico se realizó inicialmente con un examen coprológico en fresco en solución salina y con lugol, con objetivo de $40 \mathrm{X}$ en microscopio de luz, para observar la pared de los ooquistes (figura 1). Una vez que estuvieron secas las materias fecales en extendidos sobre portaobjetos, se colorearon con el método de ZiehlNeelsen modificado (9) para demostrar al microscopio, con objetivos de 40X y 100X, la presencia de las estructuras ácido-resistentes propias de este grupo de parásitos (figura 2). En estas mismas preparaciones se hizo un recuento de ooquistes en 200 campos microscópicos con objetivo de 40X y la medición con ocular milimetrado (Cambridge Instruments, Watertown, MA). Los ooquistes de Cyclospora son más grandes que los de Cryptosporidium, lo cual es básico para su clasificación. Se consideró que los primeros miden de 8 a $10 \mu$ y los segundos de 4 a $5 \mu$ de diámetro $(3,8)$.

Los parásitos de todos los pacientes se observaron al microscopio de epifluorescencia con filtro excitador de 330-380 nm, para confirmar la autofluorescencia de los ooquistes (figura 3), lo cual ayuda a su identificación $(3,8,10)$. Otras 


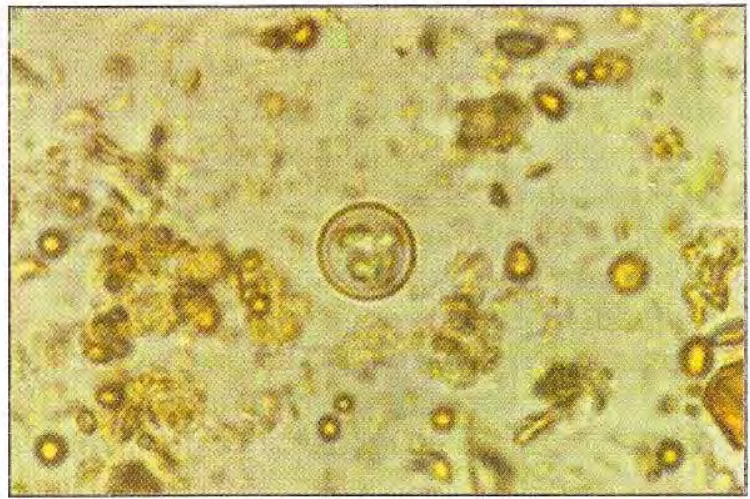

Figura 1. Preparación en fresco de muestra de heces donde se encuentra un ooquiste de Cyclospora sp., 100X.

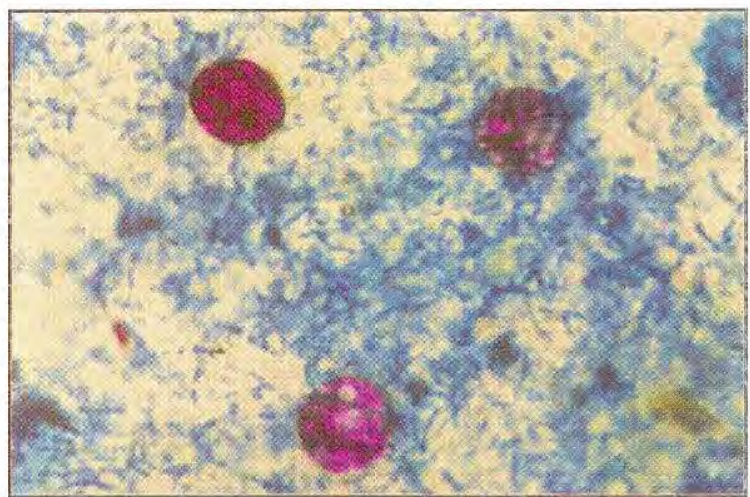

Figura 2. Muestras de heces con ooquistes de Cyclospora sp., Ziehl-Neelsen. 100X.

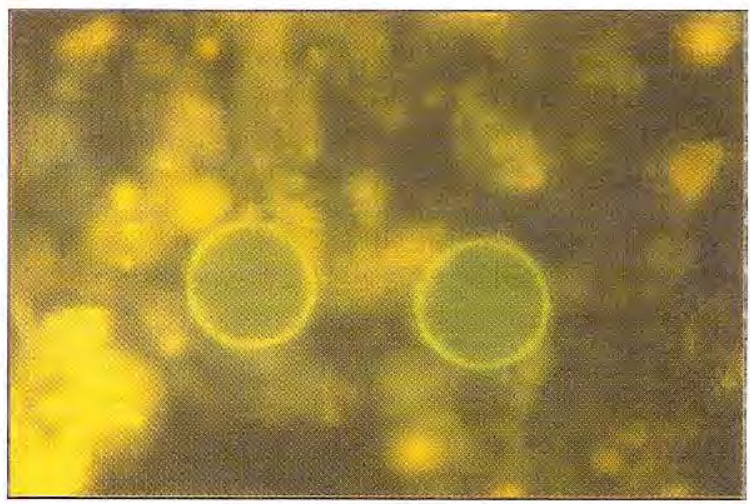

Figura 3. Observación de Cyclospora sp., luz fluorescente, $100 \times$. preparaciones se trataron con secador de punto crítico con $\mathrm{CO}_{2}$ (Hitachi HCP-2) y, luego, se cubrieron con 120 Angström de oro paladio en un cobertor iónico (Hitachi E-101). La observación se hizo con microscopio electrónico de barrido, Hitachi S-510, a 25 kilovatios (figura 4a).

\section{Resultados}

Las descripciones de las historias clínicas y sus respectivos hallazgos de laboratorio se presentan a continuación.

Caso 1. Se trata de una paciente de 31 años, de sexo femenino, con un peso de $56 \mathrm{~kg}$, residente en Medellín. Comenzó con un cuadro abrupto de diarrea acuosa intensa y malestar general, sin otros síntomas concomitantes. Después de presentar esta diarrea por una semana, tratada con remedios caseros, consultó a nuestra institución. Al realizarse el examen físico, se encontró una paciente hidratada a pesar de la diarrea, afebril, con presión arterial de 120/80 mm $\mathrm{Hg}$, pulso de $75 / \mathrm{min}$ y sin otras alteraciones clínicas.

El examen coprológico no informó la presencia de parásitos intestinales, solamente se observaron cristales de Charcot-Leyden. El estudio de azúcares no mostró alteracion alguna y el coprocultivo fue negativo. A los 29 días de iniciada la enfermedad, se le hicieron estudios hematológicos y de química sanguínea, cuyos resultados estuvieron dentro de los límites normales. La prueba de ELISA para VIH fue negativa. Se realizó un nuevo coprológico en el cual se observaron unas estructuras redondeadas de pared gruesa semejantes a Cryptosporidium, en el examen en fresco, pero de mayor tamaño. La coloración de Ziehl-Neelsen reveló estructuras ácido-resistentes, rojizas, de 8 a $10 \mu$ de diámetro, con un recuento de 25 ooquistes por 200 campos con objetivo de $40 X$. Al microscopio de luz ultravioleta se observaron los ooquistes autofluorescentes que se clasificaron como Cyclospora sp. La paciente fue sometida a tratamiento con trimetoprim-sulfametoxazole con una dosis de $800 \mathrm{mg}$ de la sulfa más $160 \mathrm{mg}$ de trimetoprim, durante 7 días. A los tres días de iniciado el tratamiento, los síntomas cesaron y el examen coprológico y las coloraciones para Cyclospora fueron negativos. 
Caso 2. Se trata de una paciente de 24 años, de sexo femenino, con peso de $46 \mathrm{~kg}$, residente en Medellín. La paciente consultó por diarrea de 15 días de evolución, algunas veces intensa, con más de 10 deposiciones al día. Al realizar el examen físico, se encontró una paciente en buen estado general, sin enfermedades concomitantes, con presión arterial de 120/80 mm Hg y pulso de $75 /$ min. No presentaba signos anormales, excepto una sensibilidad aumentada a la presión abdominal. Los exámenes de laboratorio de hematología, química sanguínea y orina fueron normales y la prueba de ELISA para VIH, negativa. El examen coprológico directo no reveló la presencia de parásitos. Con la coloración de ZiehlNeelsen, se observaron ooquistes ácidoresistentes de color rojizo que median de 8 a $10 \mu$ de diámetro, en cantidad de 4 ooquistes por 200 campos con objetivo de 40X. En las preparaciones vistas al microscopio de fluorescencia, se observaron autofluorescentes. Los ooquistes se clasificaron como Cyclospora sp. La paciente fue tratada con un medicamento antiparasitario experimental con acción para protozoos intestinales, la nitazoxanida, que tiene una presentación en tabletas de $500 \mathrm{mg}$; se le administraron 3 tabletas/ día durante 14 días. Los cinco exámenes coprológicos realizados periódicamente en los dos meses siguientes siempre fueron negativos. Los síntomas desaparecieron desde el inicio del tratamiento. Un año después, la paciente continuaba asintomática y con examen coprológico negativo para parásitos.

Caso 3. Se trata de una paciente de 33 años, de sexo femenino y residente en Medellin. Consultó por un cuadro clínico de 10 días de evolución consistente en dolor abdominal intenso de localización difusa y diarrea líquida en las noches, solamente en los dos últimos días. La paciente refirió que sentía dolor abdominal desde hacía 6 meses, algunas veces náuseas, sin vómito ni diarrea hasta hacía 10 días, cuando le habían hecho el diagnóstico de gastritis tratada con ranitidina. A partir de una endoscopia se le aisló Helycobacter pylori. En el momento de su última consulta, se encontró una paciente en aparentes buenas condiciones generales, afebril e hidratada, con presión arterial de $110 / 80 \mathrm{~mm} \mathrm{Hg}$ y dolor a la palpación superficial y profunda en la región periumbilical. El resto del examen físico fue normal, así como los exámenes de laboratorio de hematología y química sanguínea. La prueba de ELISA para detectar $\mathrm{VIH}$ también fue negativa. AI realizar el examen coprológico, se encontraron estructuras de pared gruesa semejantes a Cryptosporidium pero de mayor tamaño. Al colorearlas con Zielh-Neelsen se observaron las formas redondeadas ácido-resistentes de 8 a 10 $\mu$ de diámetro, con un recuento de 63 ooquistes por 200 campos con objetivo de 40X que, en el microscopio de fluorescencia mostraron autofluorescencia. El parásito se clasificó como Cyclospora sp.

A la paciente se le inició tratamiento con trimetoprim-sulfametoxazole en dosis de $800 \mathrm{mg}$ de la sulfa más $160 \mathrm{mg}$ de trimetroprim, durante 7 días. Los controles después del tratamiento no mostraron Cyclospora, pero se encontraron quistes de Entomoeba histolytica, por lo cual se le prescribió una tableta diaria de $500 \mathrm{mg}$ de teclozán durante tres días. En la última revisión, la paciente estaba en buenas condiciones y totalmente asintomática.

\section{Identificación de Cyclospora}

Las caracteríticas de los ooquistes observados fueron las siguientes: en los exámenes directos de materia fecal sin colorear, se localizaron estructuras esféricas de 8 a $10 \mu$ de diámetro, con una pared gruesa y cuerpos refringentes en su interior (figura 1). Con la coloración de ZiehlNeelsen modificada, se observaron estructuras de igual tamaño, con el distintivo de ser ácidoresistentes, semejantes a Cryptosporidium (figura 2), pero con diferentes tonalidades de rojo, desde rosado hasta blanco, características que son propias de los ooquistes de Cyclospora sp. $(3,4)$.

La característica de la autofluorescencia se observó al microscopio de epifluorescencia con luz ultravioleta Nikon (figura 3), técnica descrita por varios autores (3-5). Los parásitos se procesaron para verlos con gran aumento al microscopio electrónico de barrido (figura $4 a$ ), bajo el cual mostraron una pared gruesa que no permitió visualizar estructuras intracelulares. Se compararon estas formas con los quistes de 

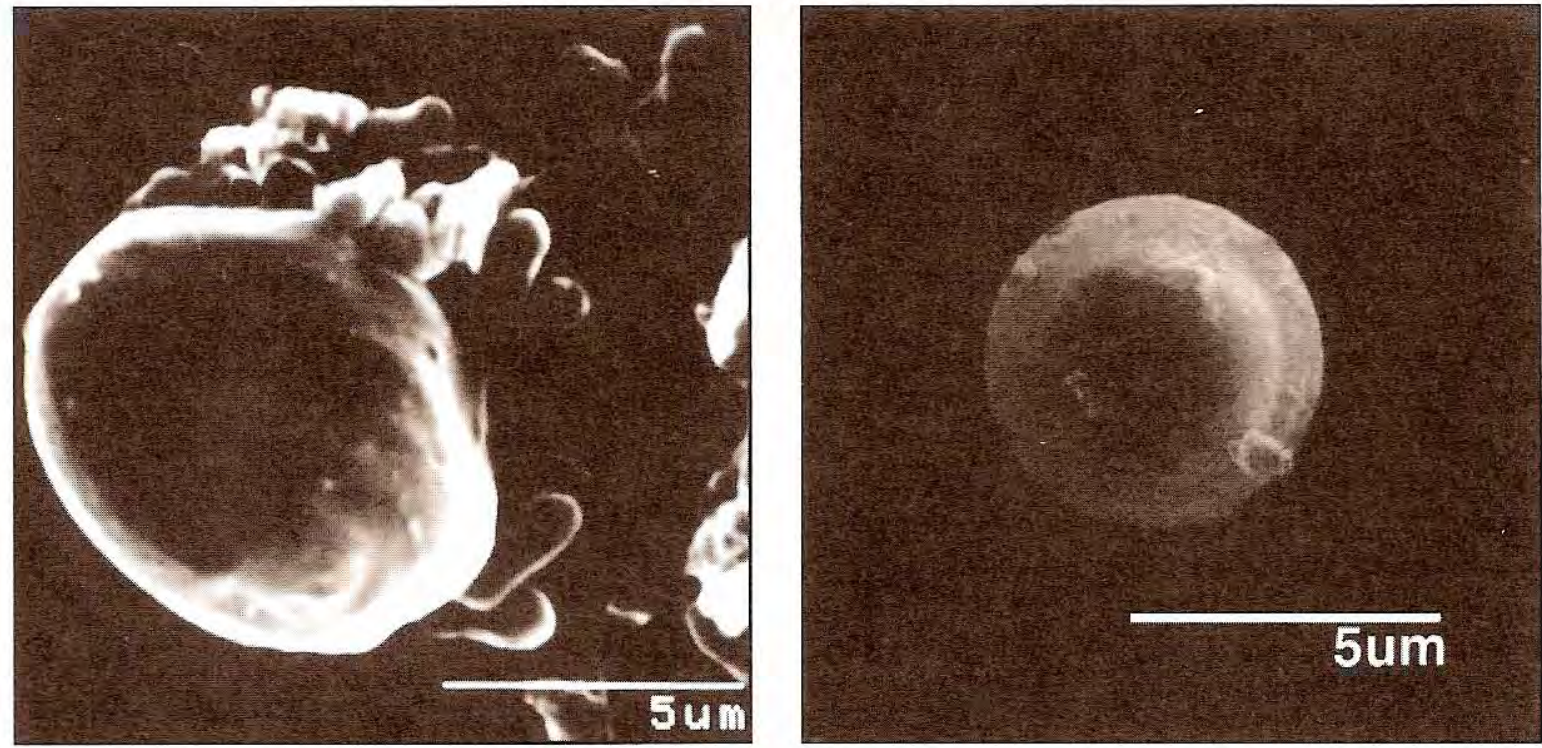

Figura 4. Ooquistes, microscopía electrónica de barrido: a) Cyclospora sp., 15.000 aumentos; se observan bacterias adheridas a su superficie; b) Cryptosporidium sp., 6.000 aumentos.

Cryptosporidium (figura $4 \mathrm{~b}$ ) y fue evidente la diferencia en forma y tamaño.

Los medicamentos empleados para el tratamiento de los tres casos fueron efectivos. No se presentaron efectos secundarios, inclusive a la nitazoxanida, un 5-nitrotiazol, que estaba en experimentación en este Instituto. Los ooquistes desaparecieron de las materias fecales al terminar el tratamiento.

\section{Discusión}

Desde el advenimiento del síndrome de inmunodeficiencia adquirida, los protozoos intestinales formadores de esporoquistes como Cryptosporidium, Isospora, Cyclospora y Microspora, se han descrito como causa de patología intestinal tanto en los pacientes con alteraciones de la inmunidad como en personas inmunocompetentes $(1,2)$.

En 1986, Soave y colaboradores informaron sobre varios pacientes inmunocompetentes que presentaron un síndrome diarreico a su regreso de una visita a México y Haití (3). En la materia fecal de estos pacientes, se observaron estructuras de 8 a $9 \mu$ de diámetro y los investigadores insinuaron que podía tratarse de un nuevo patógeno. Posteriormente, en 1990, Hart y colaboradores sugirieron la posibilidad de que en efecto fuera un nuevo patógeno cuando lo encontraron en pacientes con sida que tenían diarrea crónica (5).

La existencia del parásito se sospechó desde 1985 cuando se estudiaban varios grupos de niños con diarrea crónica en los suburbios de Lima, Perú, buscando ooquistes de Cryptosporidium en las materias fecales teñidas con la coloración modificada de Ziehl-Neelsen y el uso de anticuerpos monoclonales marcados con fluoresceína. La prevalencia del nuevo parásito varió entre 6 y 18\% (4). En 1994, cuando Ortega y colaboradores describieron el parásito como Cyclospora cayetanensis (10), se confirmó que en el intestino existían formas intraepiteliales del parásito en su estado asexual en las biopsias de los pacientes sintomáticos (9).

Al revisar los numerosos casos descritos en muchos países $(3,8,17-23)$, se concluye que los grupos de personas que tienen más riesgo de contraer la infección, son los turistas o residentes extranjeros en países en desarrollo, los agricultores y los pacientes inmunodeprimidos, principalmente con sida. La mayoría de los estudios tienden a incriminar el agua como ruta 
de infección, como se demostró en la epidemia de Chicago notificada en 1990, en la que se implicó la exposición a aguas contaminadas mantenidas por mucho tiempo en un tanque como la fuente de la infección humana (24). Igual observación se hizo en el estudio de casos y controles realizado en soldados británicos de un destacamento militar en Pokhara, Nepal (25). Estos pacientes con Cyclospora habian ingerido agua de un tanque de donde posteriormente se recobró el parásito. La incidencia de infección por este agente en repatriados residentes en Nepal fue de $7 \%$. Estos mismos autores sugieren que el tratamiento del agua con cloro puede no ser suficiente para proteger a las personas de sufrir la infección (25). Otros brotes con este parásito se informaron en Estados Unidos y Canadá, en donde se sugiere que la infección fue el resultado del consumo de vegetales o frutas contaminadas $(26,27)$. En Colombia, no se encontraron publicaciones que informaran casos de la infección. Este informe hace referencia a tres casos de personas inmunocompetentes, pero con síntomas de diarrea crónica.

El intestino delgado es la localización del parásito en el huésped humano; allí, se observa un ciclo asexual, lo que puede sugerir que sólo requiere de un huésped. Hasta el momento, no hay evidencia de que se requiera un huésped intermediario (28). En estudios por endoscopia, se han identificado zonas de mucho eritema en el duodeno distal. En las biopsias del duodeno y yeyuno, se observan diferentes grados de atrofia de las vellosidades intestinales e hiperplasia de las criptas; en la materia fecal, cuando se observan los parásitos, hay ausencia de leucocitos y eritrocitos $(29,30)$.

La diarrea crónica es la principal característica clínica en los niños y adultos que tienen el parásito. $(5,9,31)$. En Nepal, la duración de los episodios diarreicos fue de 43 días en promedio, con un rango de 4 a 107 días (18). Nuestros casos presentaron diarrea de 7 a 15 días de duración y los episodios se iniciaron súbitamente con diarrea acuosa, con 3 a 7 deposiciones diarias. Se han descrito otros síntomas asociados, como mialgias, dolores abdominales, flatulencia y náuseas. Cuando los pacientes no consultan tempranamente, la diarrea persiste por varias semanas; se puede dar la remisión espontánea de la diarrea, aunque también ocurren recaídas. Cuando dura varias semanas, hay pérdida de peso y el cuadro clínico es semejante al provocado por otros parásitos intestinales como Cryptosporidium o Giardia. En los pacientes con sida, es frecuente la diarrea crónica y la disminución del peso corporal. Bellagra y colaboradores han informado casos de pacientes con sida y coinfección de Cryptosporidium sp. y Cyclospora sp. (32).

En el laboratorio, la presencia de los ooquistes de Cyclospora se puede sospechar a partir del examen directo en solución salina y se confirma el diagnóstico con extendidos coloreados por el método de Ziehl-Neelsen, en los que se observan ooquistes de 8 a $10 \mu$ de diámetro que se tiñen de rojo, aunque no es posible una adecuada visualización de los esporozoítos. Aunque esto no es esencial para hacer el diagnóstico, es posible concentrarlos por el método de formol-éter. La autofluorescencia con que se ven los ooquistes en el microscopio de fluorescencia ayuda bastante al diagnóstico (9). La biopsia yeyunal teñida con hematoxilina-eosina muestra la inflamación con abundante infiltrado linfocitario de la lámina propia y un incremento de los linfocitos intraepiteliales donde se localizan los ooquistes. Con el microscopio electrónico de transmisión, se observa que el ooquiste inmaduro tiene una estructura fibrilar de $63 \mathrm{~nm}$; debajo de ésta hay una pared celular de $50 \mathrm{~nm}$ de grueso y el citoplasma contiene gránulos claros y oscuros. $\mathrm{El}$ ooquiste maduro tiene una envoltura fibrilar y pared similar al anterior y permite observar los esporozoítos (9). Con el microscopio electrónico de barrido, sólo se aprecia la estructura externa.

El tratamiento más utilizado es el trimetoprimsulfametoxazole que se ha empleado también para profilaxis. Esta asociación de medicamentos se considera el tratamiento de elección para Cyclospora y, además, se utiliza en los pacientes con sida en el tratamiento de la isosporosis y toxoplasmosis $(33,34)$, pero no es efectivo para Cryptosporidium $(35,36)$. La nitazoxanida, un medicamento reciente ya comercializado en México, útil contra protozoos y helmintos intestinales, fue efectiva en el único paciente al cual se le administró para Cyclospora; también 
se considera promisoria en el tratamiento de criptosporidiosis $(37,38)$.

\section{Agradecimientos}

Al biólogo Alveiro Acevedo del Departamento de Biología de la Universidad de Antioquia, por el estudio de microscopio electrónico de barrido.

\section{Referencias}

1. Botero D, Restrepo M. Otros protozoos intestinales. En: Botero D, Restrepo M, Parasitosis humanas. Tercera edición. Medellín: Editorial CIB; 1998. p.69-74.

2. Goodgame RW. Understanding intestinal spore-forming protozoa: Cryptosporidia, Microsporidia, Isospora, and Cyclospora. Ann Intern Med 1996;124:429-41.

3. Soave R, Dubey JP, Ramos LJ, Tummings M. A new intestinal pathogen? Clin Res 1986;34:533A.

4. Naranjo J, Sterling CR, Gilman R. Cryptosporidium muris-like objects from fecal samples of Peruvians. Abstracts, 38th Annual Meeting of the American Society of Tropical and Hygiene, Honolulu, December 10-14, 1989.

5. Hart AS, Ridinger MT, Soundarajan R, Peters CS, Swiantlo AL, Kocka FE. Novel organisms associated with chronic diarrhoea in AIDS. Lancet 1990;335:16970 .

6. Long EG, Ebrahimzadeh A, White EH, Swisher B, Callaway CS. Alga associated with diarrhea in patients with acquired immunodeficiency syndrome and in travelers. J Clin Microbiol 1990;28:1101-4.

7. Center for Disease Control. Outbreak of diarrhoeal illness associated with a Cyanobacteria (blue-green algae)-like bodies, Chicago and Nepal, 1989 and 1990. MMWR 1991;40:325-7.

8. Long EG, White EH, Carmichael WW, Quinlisk PM, Raja R, Swisher BL, et al. Morphologic and staining characteristics of a cyanobacterium-like organism associated with diarrhea. J Infec Dis 1991;164:199202.

9. Ortega YR, Sterling, CR, Gilman RH, Cama VA, Díaz FC. Cyclospora species: a new protozoan pathogen of humans. N Eng J Med 1993;328:1308-12.

10. Ortega YR, Gilman RH, Sterling CR. A new coccidian parasite (Apicomplexa: Eimeriidae) from humans. J Parasitol 1994;80:625-9.

11. Relman DA, Schmidt TM, Gajadhar A, Sogin M, Cross J, Yoder K, et al. Molecular phylogenetic analysis of Cyclospora, the human intestinal pathogen, suggests that it is closely related to Eimeria species. $J$ Infect Dis 1996;173:440-5.

12. Smith HV, Paton CA, Girdwood RWA, Mtambo MMA. Cyclospora in non-human primates. Vet Rec 1996;138:528.
13. García-López HL, Rodríguez-Tovar LE, Medina de la Garza CE. Identification of Cyclospora in poultry. J Emerg Infect Dis 1996:2:356-7.

14. Zerpa R, Uchima N, Huicho L. Cyclospora cayetanensis associated with watery diarrhoea in Peruvian patients. Am J Trop Med Hyg 1995;98:325-9.

15. Yai LE, Bauab AR, Hirschfeld MP, de Oliveira ML, Damaceno JT. The first two cases of Cyclospora in dogs, Sao Paulo, Brazil. Rev Inst Med Trop Sao Paulo 1997:39:177-9.

16. Eberhard ML, da Silva J, Lilley BG, Pieniazek NJ. Morphologic and molecular characterization of new Cyclospora species from Ethiopian monkey:C. cercopithecis sp.n., C. colobi sp.n., and C. papionis sp.n. J Emerg Infect Dis 1999;5:651-6.

17. Chiodini PL. A 'new' parasite: human infection with Cyclospora cayetanensis. Trans R Soc Trop Med Hyg 1994;88:369-71

18. Shlim DR, Cohen MT, Eaton M, Rajah R, Long EG, Ungar BL. An alga-like organism associated with an outbreak of prolonged diarrhea among foreigners in Nepal. Am J Trop Med Hyg 1991;45:383-9.

19. Connor BA, Shlim DR, Scholes JV, Rayburn JL, Reidy J, Rajah R. Pathogenic changes in the small bowel in nine patients with diarrhoea associated with a coccidia-like body. Ann Intern Med 1993;119:377-82

20. Bendall RP, Lucas S, Moody A, Tovey G, Chiodini PL. Diarrhoea associated with cyanobacterium-like bodies: a new coccidian enteritis of man. Lancet 1993;341:590-2.

21. Gascon J, Corachan M, Valls ME, Gene A, Bombi JA. Cyanobacterium body (CLB) in travellers with diarrhoea. Scand J Infect Dis 1993;25:253-7.

22. Markus MB, Frean JA. Occurrence of human Cyclospora infection in sub-Saharan Africa. South African Med J 1993;83:662-3.

23. Ashford RW. Ocurrence of an undescribed coccidian in man in Papua, New Guinea. Ann Trop Med Parasitol 1979;497-500.

24. Huang P, Weber JT, Sosin DM, Griffin PM, Long EG, Murphy JJ, et al. The first reported outbreak of diarrheal illness associated with Cyclospora in the United States. Ann Intern Med 1995;123:409-14.

25. Rabold JG, Hoge CW, Shlim DR, Kefford C, Rajah R, Echeverria P. Cyclospora outbreak associated with chlorinated drinking water. Lancet 1994;344:1360-1.

26. Center for Disease Control. Outbreaks of Cyclospora cayetanensis infections, United States, 1996. MMWR 1996;45:549-51.

27. Center for Disease Control. Outbreaks of Cyclospora cayetanensis infection, United States and Canada. MMWR 1996;445:611-2.

28. Sun T, llardi CF, Asnis D, Bresciani AR, Goldenberg $\mathrm{S}$, Roberts B, et al. Light and electron microscopic 
identification of Cyclospora species in the small intestine. Evidence of the presence of asexual life cycle in human host. Am J Clin Pathol 1996;105:216-20.

29. Coonor BA, Shlim DR, Scholes JV, Rayburn JL, Reidy J, Rajah R. Pathologic changes in the small bowel in nine patients with diarrhea associated with a coccidialike body. Ann Intern Med 1993;119:377-82.

30. Wurtz R. Cyclospora: a newly identified intestinal pathogen of humans. Clin Infect Dis 1994;18:620-3.

31. Berlin OGW, Novak SM, Porschen RK, Long EG, Stelma, GN, Schaeffer FW. Recovery of Cyclospora organisms from patients with prologed diarrhea. Clin Infec Dis 1994;18:606-9.

32. Bellagra N, Ajana F, Coignard C, Caillaux M, Mouton Y. Co-infections with Cryptosporidium $\mathrm{sp}$. and Cyclospora sp. in an AIDS stage HIV patient. Ann Biol Clin (Paris) 1998;56:476-8.

33. Shlim DR, Pandey P, Rabold JG, Walch A, Rajah R. An open trial of trimethoprim alone against Cyclospora infections. J Travel Med 1997;4:44-5.
34. Pape JW, Verdier RI, Boncy M, Boncy J, Johnson WD. Cyclospora adults infected with HIV. Clinical manifestations, treatment, and prophylaxis. Ann Intern Med 1994;121:654-7.

35. Madico G, Gilman RH, Miranda E, Cabrera L, Sterling CR. Treatment of cyclospora infections with co-trimoxazole. Lancet 1993;342:122-3.

36. Booy R, Tudor-Williams G. Co-trimoxazole for Cyclospora infection. Lancet 1995;345:1303-4.

37. Doumbo O, Rossignol JF, Pichard E, Traore HA, Dembele TM, Diakite $M$, et al. Nitazoxanide in the treatment of cryptosporidial diarrhea and other intestinal parasitic infections associated with acquired immunodeficiency syndrome in tropical Africa. Am J Trop Med Hyg 1997:56:637-9.

38. Rossignol JF, Hidalgo $H$, Feregrino $M$, Higuera WH, Gomez JL, Romero J, et al. A double-blind placebocontrolled study of nitazoxanide in the treatment of cyptosporidial diarrhoea in AIDS patients in Mexico. Trans R Soc Trop Med Hyg 1998;92:663-6. 